\title{
A ATUAÇÃO DO ENFERMEIRO NA ATENÇÃO PRIMÁRIA À SAÚDE REFERENTE À CLASSIFICAÇÃO DE RISCO ATRAVÉS DO SISTEMA DE TRIAGEM DE MANCHESTER.
}

\section{NURSES 'ACTIVITY IN PRIMARY HEALTH CARE REGARDING RISK CLASSIFICATION THROUGH MANCHESTER TREATMENT SYSTEM}

\author{
Martha Honorato da Silva \\ Enfermeira, Especialista em Programa de Saúde da Família \\ Marcia Maria Carvalho Pimentel \\ Enfermeira, Especialista em Programa de Saúde da Família \\ Lucas Tavares Nogueira \\ *** Enfermeiro, Especialista em Gestão Pública
}

Daniel de Azevedo Teixeira

Farmacêutico-Bioquímico, Mestre em Imunopatologia e Doutorando em Biocombustíveis

\section{Resumo}

A Atenção Primária à Saúde (APS) caracteriza-se como conjunto de intervenções de saúde no âmbito individual e coletivo que envolve não somente o indivíduo como sua família, buscando a promoção, prevenção, diagnóstico, tratamento e reabilitação. O protocolo de classificação de risco de Manchester ou Sistema de Triagem de Manchester (STM) é uma metodologia que vem sendo utilizada amplamente no Brasil, desenvolvida na Inglaterra, na cidade de Manchester, com o objetivo de viabilizar o atendimento dos pacientes em nível primário. O presente estudo aborda em caráter bibliográfico a importância da atuação do enfermeiro na atenção primária utilizando o Sistema de Triagem de Manchester.

Palavras chave: Enfermeiro, Protocolo de Manchester, Atenção Primária.

\begin{abstract}
Primary Health Care (PHC) is characterized as a set of individual and collective health interventions that involve not only the individual as his family, seeking promotion, prevention, diagnosis, treatment and rehabilitation. The Manchester risk classification protocol or Manchester Screening System (STM) is a methodology that has been widely used in Brazil, developed in England in the city of Manchester with the aim of making possible the care of patients at primary level. The present study approaches in bibliographical character the importance of the nurses' performance in primary care using the Manchester Triage System.
\end{abstract}

Key words: Nurse, Manchester Triage System, Primary Health Care 


\section{Introdução}

O Sistema Único de Saúde (SUS) é formado pelo conjunto de todas as ações e serviços de saúde prestados por órgãos e instituições públicas, federais, estaduais e municipais, da administração direta e indireta e das funções mantidas pelo poder público. (BRASIL, 2000). Nesse sentido, o SUS é uma política pública de saúde que assume e consagra os princípios da Universalidade, Equidade e Integralidade da atenção à saúde da população brasileira, capaz de garantir o acesso universal da população a bens e serviços que garantam sua saúde e bemestar, de forma equitativa e integral (TEIXEIRA, 2011).

A Atenção Primária à Saúde (APS) caracteriza-se como conjunto de intervenções de saúde no âmbito individual e coletivo que envolve não somente o indivíduo como sua família, buscando a promoção, prevenção, diagnóstico, tratamento e reabilitação. É desenvolvida por meio do exercício de práticas gerenciais e sanitárias, democráticas e participativas, sob a forma de trabalho em equipe, dirigidas às populações de territórios bem delimitados. Utilizam-se tecnologias de elevada complexidade e baixa densidade, que devem atender aos problemas de saúde de maior frequência e relevância da população. Neste sentido, a APS deve considerar o sujeito em sua singularidade, complexidade, integralidade e inserção sócia cultural e buscar a promoção de sua saúde, a prevenção e tratamento de doenças e a redução de danos ou de sofrimentos que possam estar comprometendo sua saúde (CONASS, 2004; BRASIL, 2006; GÉRVAS; FERNÁNDEZ, 2009).

Composta por uma equipe com caráter multidisciplinar e multiprofissional, como, por exemplo, o enfermeiro, o médico, auxiliar de enfermagem e agente comunitário de saúde. Destacam-se as ações do enfermeiro, que estão pautadas no gerenciamento da unidade e assistência à comunidade. Assistência esta que é pautada através dos sinais e sintomas apresentados pelo paciente com a implantação do acolhimento com classificação de risco na APS; essas práticas assistenciais tornam-se mais reestruturadas, por se tratar de instrumento que facilita o fluxo de paciente que procura os serviços de saúde gerando um atendimento resolutivo e humanizado. Essa Classificação de Risco é definida como um processo dinâmico de identificação dos pacientes 
que necessitam de tratamento, de acordo com o potencial de risco, agravos à saúde ou grau de sofrimento (SERVIN et al, 2010).

O protocolo de classificação de risco de Manchester ou Sistema de Triagem de Manchester (STM) é uma metodologia que vem sendo utilizada amplamente no Brasil, desenvolvida na Inglaterra na cidade de Manchester. Tal metodologia é estruturada por fluxogramas que representam as principais queixas apresentadas, definindo o atendimento pela prioridade clínica e não por ordem de chegada. $O$ paciente pode ser classificado em cinco níveis de prioridade que representam desde demandas emergentes às demandas não urgentes. Para cada nível é estabelecido uma cor e um tempo alvo para atendimento médico e para reavaliação do enfermeiro (MACKWAY-JONES, 2010 citado por SOUZA, et al. 2013).

O enfermeiro é o profissional indicado pelo Ministério da Saúde para o devido processo de classificação de risco, devendo receber treinamentos específicos utilizando protocolos pré-estabelecidos, com o objetivo de classificar por prioridades clínicas e não por ordem de chegada, reorganizando o fluxo e melhorando o atendimento humanizado (BRASIL, 2006).

A classificação é um dos primeiros atendimentos prestado pelo enfermeiro aos usuários dos serviços de urgência e emergência. Tem por finalidade a avaliação inicial, seleção e encaminhamento dos clientes às unidades/especialidades adequadas à sua assistência de acordo com os critérios estabelecidos, tendo ganhado tempo neste atendimento, que exige precisão e agilidade, além de dinamizar todo processo de assistência (AZEVEDO; BASBOSA, 2011).

Baseados nos contextos acima descritos, esse estudo objetivou discutir a atuação do enfermeiro no acolhimento com classificação de risco na Atenção Primária à Saúde, que, por sua vez, tem como finalidade realizar a classificação a todos os usuários que chegarem à unidade pela demanda espontânea, classificando o paciente a partir das queixas levantadas, de acordo com potencial de risco e agravo e não por ordem de chegada, realizando de forma humanizada, padronizando o atendimento, e organizando o serviço.

\section{Metodologia}

\section{Classificação do estudo}


O presente estudo trata-se de uma pesquisa bibliográfica, de natureza qualitativa e caráter descritivo. A pesquisa bibliográfica é um estudo sistematizado desenvolvido com base em material publicado em livros, revistas, jornais, redes eletrônicas, isto é, material acessível ao público em geral, fornecendo instrumental analítico para qualquer outro tipo de pesquisa (MORESI, 2003).

\section{Procedimentos da coleta das fontes teóricas}

Para realizar a coleta das informações foram utilizadas as bases de dados SCIELO (Scientific Electronic Library Online), LILACS (Literatura Latino-americana e do Caribe em Ciências da Saúde), BVS (Biblioteca Virtual em Saúde), e fontes como monografias, teses e buscas online. A escolha por essas bases de dados deve-se ao fato de serem bibliotecas eletrônicas que abrangem uma coleção variada de periódicos científicos.

A seleção do material referente ao uso deste estudo foi realizada através da leitura criteriosa dos resumos; leitura aprofundada dos textos adequados para 0 estudo e, finalmente, análise das informações.

A identificação dos artigos nas bases de dados eletrônicas se deu através da utilização das seguintes palavras-chave contextualizadas ao tema em estudo: Protocolo de Manchester, protocolo de classificação de risco, Enfermeiro; Enfermagem; Atenção Primária a Saúde. Além disso, foram usadas combinações de dois ou mais destes termos.

De acordo com os critérios de inclusão propostos no estudo, as publicações devem estar disponíveis na íntegra nas bases de dados indicados, voltadas ao objetivo do estudo e publicados em qualquer período.

\section{Análise e interpretação das informações}

Visando atender aos objetivos deste trabalho, a última etapa realizada nesta pesquisa bibliográfica foi a organização dos dados e a redação do relatório. Foram selecionados 56 artigos, monografias, dissertações e teses, em seguida, realizadas uma leitura criteriosa de cada uma das fontes de dados visando identificar e satisfazer os objetivos deste estudo, logo após a leitura e análise foram selecionados 40 artigos para fazer parte do conteúdo desse estudo. 


\section{Discussão}

De acordo com a Constituição da República Federativa do Brasil, de 1988 a saúde é um direito de todos e dever do Estado, sendo esse um dos grandes desafios para o SUS. Não há como negar que muitos foram os avanços do sistema de saúde pública brasileira, como o aumento da cobertura dos serviços e melhora dos indicadores. Porém, essa universalização ainda não se concretizou plenamente, podendo ser vista nas unidades de saúde de urgência e emergência muitas queixas quanto às imensas filas e demora do tempo de atendimento (BRASIL, 2006).

Reconhecendo estes problemas o governo do Estado de Minas Gerais adotou o processo de classificação de risco do protocolo de Manchester a fim de reorganizar as portas de entrada dos serviços de urgência, classificando-os pelo nível de prioridade com identificação através das cores e do tempo limite.

Após a aplicação exitosa do protocolo nas unidades de urgência/emergência do Estado de Minas Gerais expandiu-se então o uso para APS e em todas as unidades básicas do Estado, visto que muitos daqueles que aguardavam nas grandes filas de espera do serviço de urgência poderiam ter seus casos solucionados na APS.

Após a aquisição dos equipamentos a SES-MG em parceria com o canal Minas Saúde promoveu o Curso de Capacitação em Classificação de Riscos em Urgência e Emergência à Distância - STM. O curso foi oferecido aos gestores, enfermeiros e médicos para capacitá-los na implantação do protocolo nas portas de atendimento de todo o Estado. Após a capacitação dos profissionais e a sua devida certificação, só faltava a implementação efetiva da metodologia de acolhimento com classificação de risco, segundo o protocolo de Manchester.

Para Queiroz (2013) há fatores determinantes no processo de classificação como facilitadores e dificultadores, tais como:

Facilitadores:

- O aparelho disponibilizado com tela sensível ao toque e com 52 fluxogramas, o qual imprime rapidez à classificação e diminuindo o tempo gasto para classificar o paciente. 
- No caso de termos mais de um paciente que demande atendimento médico, o protocolo se mostra eficaz na priorização do atendimento.

Dificultadores:

- Segundo o STM todo usuário que procurar atendimento deverá ser classificado e encaminhado à consulta médica. $O$ que centra $O$ atendimento na figura desse profissional em detrimento das outras classes profissionais que exercem funções importantíssimas nas APS e também nos Núcleo de Assistência à Saúde da Família (NASF), tais como fisioterapeutas, psicólogos, assistentes sociais, nutricionistas, educadores físicos e odontólogos.

- Na maioria das vezes o protocolo é posto em prática e executado sem a efetiva comunicação com os demais serviços de urgência/emergência, ficando a APS sem nenhuma retaguarda para os casos mais graves.

Silva; Barros; Torres (2012) também ressaltam em seus estudos que a há fatores dificultadores e facilitadores, para a realização do protocolo, tendo como um fator dificultador a aceitação dos profissionais para implantação da classificação de risco na APS, uma vez que receiam perder a autonomia previamente conquistada nos atendimentos da demanda espontânea em realizar a consulta de enfermagem, já que a classificação se da a partir dos discriminadores do STM. Diante disso, Souza (2009) e Costa (2012) relata que o STM trouxe para o profissional enfermeiro a garantia e o respaldo do atendimento, com a tomada de decisão, com o dever de discriminar e avaliar a queixa apresentada, tendo uma autonomia de realizar o atendimento e direcionar de acordo a especialidade. Outro fator dificuldador, relatado é o não envolvimento da população no processo de implantação.

A partir desses fatores cabe ao enfermeiro responsável da unidade e pela classificação, treinar sua equipe para que estejam preparados a receber os usuários que chegarem pela demanda espontânea, orientando-os, quanto ao funcionamento do protocolo e o processo de STM. Preparar sua comunidade quanto à mudança do sistema a partir de cartazes, panfletos e palestras, para que esses pacientes possam familiarizar e entender esse novo método, não criando um sentimento de aversão à nova forma de atendimento, encarando-o como um modelo melhor e preciso para solucionar os seus casos, priorizando-o de acordo com o protocolo em estudo.

Como o protocolo de Manchester é estruturado a partir de fluxogramas, para identificar a prioridade, o enfermeiro classificador responsável pela unidade ao 
realizar este processo não deve ficar focado somente a este instrumental, durante a classificação deve-se levar em conta o sujeito com um todo, em um ser biopsicossocial, visando à integralidade, como compreende os princípios do SUS e da APS.

O acolhimento com classificação de risco é um dispositivo que opera os princípios da Política Nacional de Humanização e possui como objetivo a consecução de uma assistência com responsabilidade e vínculo, ampliando o acesso do usuário ao SUS, por meio do acolhimento resolutivo baseado em critérios de risco. Para isso é fundamental que as equipes estejam instrumentalizadas e qualificadas para identificação de risco e vulnerabilidade do usuário.

A classificação de risco é feita aos pacientes que chegarem a APS pela demanda espontânea. Essas situações não programadas podem ser acolhidas na unidade. Para Brasil (2013) é de grande importância e necessidade que os acompanhamentos dos usuários continuem a partir das agendas dos profissionais, sob a tutela da APS, conduzidos ao pronto atendimento. Além disso, é de fundamental importância que as unidades estejam abertas e preparadas para acolher o que não pode ser programado, as eventualidades e imprevistos, muitas vezes essas situações demandará continuidade no cuidado, em outras, poderá requerer, inclusive, apoio ou encaminhamento para outros serviços. Pois, o usuário não pode decidir o dia e a hora em que, por exemplo, ficará gripado ou ter uma dor de cabeça.

Queiroz (2013) ressalva que o protocolo ainda necessita de melhorias e apresenta-se ainda ineficaz na APS para uma série de casos que apresentem na unidade, nas quais o enfermeiro tem plena autonomia para solucionar a queixa, orientando o usuário ou agendando a consulta médica para outra data. Em contrapartida Souza; Barros e Torres (2012) afirmam que o protocolo possibilitará um atendimento humanizado, ágil, de acordo com as necessidades da comunidade, trazendo uma grande melhoria aos serviços.

Enfim, todos os profissionais devem estar envolvidos na implantação do acolhimento com classificação de risco, devendo orientar o fluxo, participando da discussão e da reorganização dos atendimentos da unidade.

\section{Considerações finais}


Pode-se observar que a APS tem evoluído nos últimos anos buscando cada vez mais alcançar os princípios do SUS, sendo o processo de acolhimento com classificação de risco uma metodologia que pode ser compreendida por diversos autores.

O STM foi originalmente elaborado para os serviços de urgências, ao longo do período de utilização, percebeu-se a necessidade de adequações para o uso na APS, sendo que o método de classificação proposto pelo protocolo não deve ser mudado, mantendo-se a segurança e a padronização entre os diversos pontos da rede. A classificação deve ser entendida como uma das ferramentas de avaliação dos casos com queixa clínica. O enfermeiro classificador não deve direcionar-se somente ao caso clínico do paciente, outros aspectos devem ser observados como: vulnerabilidade individual, familiar, ambiental e psicossocial.

Nos dias atuais ainda não é possível ver a utilização do processo de classificação de risco na APS devido a vários empecilhos já citados, mas que pode ser resolvido adaptando o protocolo à unidade de saúde sem que se mude a metodologia do STM. Mas, futuramente como diante das inúmeras vantagens deste serviço nas APS's, sua utilização será fundamental e viável, a fim de promover uma maior segurança técnica. Cabe ao enfermeiro responsável pelas unidades adaptarse a esse serviço mantendo-se sempre capacitado e atualizado com as novas demandas.

Diante disso, é possível afirmar que é preciso restituir no cotidiano o princípio da universalidade, e a responsabilização pela saúde dos usuários, construindo vínculos entre os profissionais e a população, objetivando-se na construção coletiva de estratégias que promovam mudanças nas práticas dos serviços de saúde e garantindo uma melhor qualidade de vida aos indivíduos.

\section{Referências}

AZEVEDO, J.M.R.; BARBOSA, M.A. Triagem em serviços de saúde: percepções dos usuários. R Enferm UERJ, Rio de Janeiro, 2007 jan/mar, p. 1-7. Disponível < http://www.facenf.ueri.br/v15n1/v15n1a05.pdf> Acesso em: 22, fev. de 2014.

BRASIL. Conselho Nacional de Secretários de Saúde. Atenção Primária e Promoção da Saúde / Conselho Nacional de Secretários de Saúde. -Brasília: CONASS, 2011. Disponível <http://www.conass.org.br/colecao2011/livro_3.pdf> Acesso em 20, abr. de 2014. 
BRASIL. Ministério da Saúde, PACTOS pela saúde, Politica Nacional de Atenção Básica, vol. 4, Brasília DF, 2006, Disponível em:

<http://bvsms.saude.gov.br/bvs/publicacoes/politica_nacional_atencao_basica_2006. pdf> Acesso em: 22,fev. de 2014.

BRASIL. Ministério da Saúde; Humaniza SUS Acolhimento com avaliação e classificação de risco: Um paradigma ético-estético no fazer em saúde.

Secretaria-Executiva; Núcleo Técnico da Politica Nacional de Humanização. BrasíliaDF, 2004. Disponível em: <

http://bvsms.saude.gov.br/bvs/publicacoes/acolhimento.pdf> Acesso em: 21, mar. de 2014.

BRASIL. Ministério da Saúde; Humaniza SUS Acolhimento e classificação de risco nos serviços de urgências. Secretaria-Executiva; Núcleo Técnico da Politica Nacional de Humanização. Brasília- DF, 2009. Disponível em: <

http://bvsms.saude.gov.br/bvs/publicacoes/acolhimento_classificaao_risco_servico_u rgencia.pdf> Acesso em: 21, mar. de 2014.

BRASIL. Programa de Agente Comunitário de Saúde PACS/ Ministério de Saúde, Secretaria Executiva- Brasília 2001. Disponível em; $<$ http://bvsms.saude.gov.br/bvs/publicacoes/pacs01.pdf> Acesso em: 17, abr. de 2014.

BRASIL. Ministério da Saúde, Secretaria de Atenção a Saúde. Acolhimento à Demanda Espontânea, Caderno de Atenção Básica. n. 28; volume 1; $1^{\circ}$ edição, Brasília- DF 2013 Disponível em: <https:

http://bvsms.saude.gov.br/bvs/publicacoes/acolhimento_demanda_espontanea_cab2 8v1.pdf > Acesso em: 09, jul. de 2014

BRASIL. Ministério da Saúde; Secretaria Executiva. Sistema Único de Saúde (SUS): Princípios e Conquistas - Brasília 2000; 44p. Disponível em < http://bvsms.saude.gov.br/bvs/publicacoes/sus_principios.pdf> Acesso em: 17, fev. de 2014.

CONASS. Conselho Nacional de Secretários de Saúde. Atenção PrimáriaSeminário do Conass para construção consensos /Conselho Nacional de Secretários de Saúde. - Brasília. 2004. 44 p. Disponível em < http://www.conass.org.br/conassdocumenta/cd_2.pdf >>. Acesso em: 22, fev. de 2014.

CONASS. Conselho Nacional de Secretários de Saúde. Atenção Primária e Promoção da Saúde / Conselho Nacional de Secretários de Saúde. - Brasília 2007. Disponível em: < http://bvsms.saude.gov.br/bvs/publicacoes/colec_progestores_livro8.pdf> Acesso em: 10, mar. de 2014.

COREN-MG. Conselho Regional de Enfermagem de Minas Gerais. PARECER TÉCNICO №. 136 / 2011. Disponível em: < http://www.corenmg.gov.br/anexos/Parecer\%20T\%C3\%A9cnico\%20136.2011.pdf> Acesso em: 22 mar. de 2014. 
COSTA, J.M.C. Relato de experiência da Classificação de risco em UBS Centro de Saúde Marivanda Baleeiro. UFMG, Lagoa Santa MG, 2012. Disponível em: <https://www.nescon.medicina.ufmg.br/biblioteca/imagem/3893.pdf> Acesso em: 23 maio. de 2014.

COUTINHO.A.A.P; CECÍLIO.L.C.O; MOTA.J.A.C. Classificação de risco em serviços de emergências: uma discussão da literatura sobre o Sistema de Triagem de Manchester. UFMG, Belo Horizonte, Brasil. 2012. Disponível em: < file:///C:/Users/home/Downloads/v22n2a10.pdf> Acesso em: 14, fev. de 2014.

CONILL,E.M; Ensaio histórico-conceitual sobre a Atenção Primária à Saúde: desafi os para a organização de serviços básicos e da Estratégia Saúde da Família em centros urbanos no Brasil. Cad. Saúde Pública, Rio de Janeiro, 24 Sup 1:S7-S27, 2008 Disponível em: < http://www.scielo.br/pdf/csp/v24s1/02.pdf> Acesso em: 06, mar. de 2014.

GÉRVAS.J; PÉREZ.M; FERNÁNDEZ.M.P. Uma Atenção Primária forte no Brasil: Como fortalecer os acertos e corrigir as fragilidades da estratégia de saúde da família.

Equipe CESCA, Madrid, Espanha, 2009. Disponível em: < http://www.sbmfc.org.br/media/file/documentos/relatoriofinal_portugues.pdf> Acesso em: 12, mar. de 2014.

MARTINS, C.C. "Análise do processo de acolhimento em Unidades Básicas de Saúde de Minas Gerais tendo por referência o Plano Diretor da Atenção Primária à Saúde". Dissertação de Mestrado Modalidade Profissional em Saúde Pública; Fundação Oswaldo Cruz, FIOCRUZ, Ministério da Saúde. Rio de Janeiro jun. 2011. Disponível: < file:///C:/Users/home/Downloads/martinsccm.pdf> Acesso em: 12 de mar. de 2014.

MATTA, G.C; MOROSINI, M.V.G . Dicionário Profissional em Saúde. Atenção Primária, Fundação Oswaldo Cruz, Escola técnica de Saúde Joaquim Venâncio, Rio de Janeiro 2009. Disponível em: <

http://www.epsjv.fiocruz.br/dicionario/verbetes/ateprisau.html> Acesso em :20, abr. de 2014.

MEDONÇA. M.H.M; VASCONCELLOS, M.M; VIANA, A.L.A. Atenção Primária à Saúde no Brasil, Saúde Pública, Rio de Janeiro, 24 Sup 1:S4-S5, 2008. Disponível <http://www.scielo.br/pdf/csp/v24s1/01.pdf> Acesso em: 14, abr. de 2014.

MENDES, E.V. O Cuidado das Condições Crônicas na Atenção Primária à Saúde: O Imperativo da Consolidação da Estratégia da Saúde da Família. Brasília, Organização Pan- Americana de Saúde - 2012. 512p. Disponível em: < http://bvsms.saude.gov.br/bvs/publicacoes/cuidado_condicoes_atencao_primaria_sa ude.pdf> Acesso em: 12, mar. de 2014.

MORESI, E. Metodologia da pesquisa, Universidade Católica de Brasília, BrasíliaDF 2003. Disponível em: < 
http://ftp.unisc.br/portal/upload/com_arquivo/1370886616.pdf > Acesso em: 05 de jun. de 2014.

OLIVEIRA, G. S; MENEGUCI, L. R. Os usuários de um pronto atendimento reconhecem o que é urgência e emergência? Contribuição ao protocolo de classificação de risco. Varginha - MG, 2009. Disponível em:

<file://C:/Users/home/Downloads/TCC\%20Classifica\%C3\%A7\%C3\%A30\%20de\%20 Risco.pdf> Acesso em: 23, mar. de 2014.

QUEIROZ, R.L.B. O uso do protocolo de Manchester na Atenção Básica e suas implicações. UFMG Governador Valadares, 2013. Disponível em: < https://www.nescon.medicina.ufmg.br/biblioteca/imagem/4188.pdf> Acesso em: 28, mar. de 2014.

RAMOS, C.M.O. O cotidiano do acolhimento com classificação de risco no serviço de emergência: Contribuições para gerencia de enfermagem. Dissertação de Mestrado, Escola de Enfermagem Anna Nery- UFRJ, 2011. Disponível em: < http://teses2.ufrj.br/51/dissert/EEAN_M_CamillaMariaDeOliveiraRamos.pdf > Acesso em: 13, fev. de 2014.

SOUZA,R.S. BASTOS,M.A.R. Acolhimento com classificação de Risco: 0 processo vivenciado pelo profissional enfermeiro. Relato de experiência;:;12(4): 581-586, out./dez., 2008. Disponível em:

<file://C:/Users/home/Downloads/v12n4a18.pdf> Acesso em: 03, abril, de 2014.

SERVIN, SANTIAGO, C. N et al. Protocolo de classificação de risco. Politica nacional de humanização. Prefeitura de São luiz do Maranhão 2010 disponível em:

<http://bvsms.saude.gov.br/bvs/publicacoes/protocolo_acolhimento_classificacao_ris co.pdf> Acesso em: 25, fev. de 2014.

STARFIELD. B. Atenção Primária: equilíbrio entre as necessidades de saúde, serviços e tecnologia. Brasília- UNESCO, Ministério da Saúde, 2002, 726p.

Disponível em:<

http://bvsms.saude.gov.br/bvs/publicacoes/atencao_primaria_p1.pdf> Acesso em: 10, mar. de 2014.

SILVA, P.M. BARROS, K.P. TORRES, H.C. Acolhimento Com Classificação de Risco na Atenção Primária: Percepção dos Profissionais de Enfermagem. remE - Rev. Min. Enferm.;16(2): 225-231, abr./jun. de 2012. Disponível em: < file:///C:/Users/home/Downloads/v16n2a11\%20(1).pdf> Acesso em: 16, jul. de 2014.

TEIXEIRA, C. Os princípios do sistema único de saúde, Salvador, Bahia, Junho 2011. Disponível em< http://www.saude.ba.gov.br/pdf/OS_PRINCIPIOS_DO_SUS.pdf >. Acesso em: 22 de fev. de 2014. 